\title{
MicroRNA-214-3p inhibits the stem-like properties of lung squamous cell cancer by targeting YAP1
}

Tingting $\mathrm{Lu}^{\dagger}$, Ying Yang ${ }^{\dagger}$, Ziming Li and Shun Lu* ${ }^{*}$

\begin{abstract}
Background: Emerging evidence reveals that microRNAs (miRNAs) play a crucial role in tumor progression, but the underlying mechanism of microRNAs in lung squamous cell cancer (LSCC) remains unclear.

Method: Western-blotting and quantitative real-time PCR (q-PCR) were carried out to detect mRNA and protein expression. Cell proliferation was evaluated by Cell Counting Kit-8 (CCK-8), colony-forming assay or sphere-forming assay, respectively.

Results: MiR-214-3p was markedly de-regulated in LSCC tissues and was inversely related to the level of Yes-associated protein1 (YAP1), which is the core transcription regulator of the Hippo signaling pathway. Kaplan-Meier survival curves illustrated that patients with high miR-214-3p expression demonstrated more favorable clinical outcomes. MiR-214-3p overexpression (OE) repressed proliferation and cancer stem-like cells (CSCs) properties in vitro and in vivo xenograft mouse model. Mechanistically, luciferase activity assay revealed that miR-214-3p directly targets YAP1 by specifically binding on the $3^{\prime}$ UTR of YAP1.
\end{abstract}

Conclusion: MiR-214-3p plays a pivotal role in CSCs properties by targeting YAP1, which provides a potential treatment strategy for LSCC patients.

Keywords: MiR-214-3p, YAP1, CSC properties

\section{Background}

Globally, lung cancer (LC) has high mortality rates [1]. Targeted drugs have been developed in lung adenocarcinoma (AC) to inhibit tumor progression, which remarkably changed treatment strategies and brought positive news to patients with EGFR mutation and ALK or ROS1 rearrangements. Compared with AC, the mainstream treatment regimens for lung squamous cell cancer (LSCC) are still platinum-containing chemotherapeutics and immune therapy, and the targeted therapy for

\footnotetext{
*Correspondence: shunlu@sjtu.edu.cn

${ }^{\top}$ Tingting Lu and Ying Yang contributed equally to this work

Department of Shanghai Lung Cancer Center, Shanghai Chest Hospital, Shanghai Jiao Tong University, 241 Huaihai West Road, Shanghai 200030,

People's Republic of China
}

advanced LSCC is rare. Therefore, it is urgent to clarify the underlying mechanism of LSCC to develop new and innovative treatment strategies for LSCC patients.

MicroRNAs are a cluster of noncoding RNAs that modulate gene expression [2]. Recently, microRNAs became known to regulate tumorigenesis. Increasing evidence has shown that aberrant miRNA expression participates in diverse pathogenic processes, such as tumor apoptosis and CSC properties [3-6]. Moreover, microRNA-controlled cancer stem cells (CSCs) have caused widespread concern as various studies link miRNAs to the characteristics of CSCs and differentiation of embryonic stem cells [7-9]. For instance, miR-205 OE remarkably decreases the proliferation of CSCs in human pancreatic cancer [10] while low expression of miR-613 contributed to the 
expansion of liver CSCs by enhancing the dedifferentiation of hepatoma cells [11].

Hippo is a traditional signaling pathway that regulates various biological processes in both drosophila and human species $[12,13]$. The Hippo pathway components involve a major kinase cascade and scaffold proteins and YAP1 is the core transcription regulator of the Hippo signaling pathway. Briefly, in Hippo pathway, Mammalian Sterile20-like Kinase1/2 (Mst1/2) forms a complex with the adaptor protein Salvador1 (Sav1) and ultimately results in YAP1 phosphorylation. Furthermore, phosphorylated YAP1 is sequestered in the cytoplasm and then degraded through the ubiquitin-proteasome [14]. Increasing evidence suggests that high levels of the YAP1 expression are present in distinct human tumors such as in colon, liver, ovarian, cholangiocyte, and prostate cancers [15-18]. Our group previously demonstrated the contribution of YAP1 to the maintenance of CSC activities in FGFR1-amplified lung squamous cell cancer [19].

In this study, to elucidate the function of miRNA in LSCC initiation and progression, we detected the selected miRNA expression profile in 20 LSCC human samples using the q-PCR (Additional file 1: Figure S1). Our results highlighted the low expression of miR-214-3p in human LSCC samples compared to adjacent normal samples. For the first time, we reported that miR-214-3p inhibited CSC capacity by targeting YAP1 in LSCC. Furthermore, we verified that miR-214-3p took part in activating the Hippo signaling activity. Altogether, we confirmed that miR-214-3p exhibited as a tumor suppressor in LSCC initiation, and it also serves as a possible target for LSCC.

\section{Materials and methods}

\section{Cell lines}

The human H520 and HCC95 cell lines were purchased from the American Type Culture Collection. MiR-214-3p and Negative Control (NC) inhibitors and mimics were from Ribobio Technology.

\section{Human tissue specimens}

For research use, the LSCC samples were collected from the Department of Oncology, Shanghai Chest Hospital. Written consent forms were obtained from every LSCC patient. The Institute Research Ethics Committee of Shanghai Chest Hospital approved all protocols.

\section{Western blotting}

YAP1(\#4912), LATS1(\#9153), p-YAP1(\#4911), Vimentin(\#12020), Snail(\#3879), and Tubulin(\#2148) antibodies were all from Cell Signaling Technology (CST, Danvers, MA). OCT4 (ab19875), CD133 (ab19898) and SOX2 (ab171380) were from Abcam. Protein was obtained from cells by using Radio-Immunoprecipitation Assay (RIPA) (Sigma-Aldrich). Equal quantities of protein lysates were loaded on the Sodium dodecyl sulfate-Polyacrylamide Gel Electrophoresis gels (SDS-PAGE gels), and gel electrophoresis was applied to transfer the protein onto a nitrocellulose membrane. The protein strips were ultimately detected by enhanced chemiluminescence (Thermo Fisher Scientific) and captured using the Odyssey Infrared Imaging Analysis Software.

\section{qRT-PCR}

Through the Trizol Reagent (Life Technologies, CA, USA), the total RNA was collected. According to the manufacturer's protocol, the q-PCR was conducted using the SYBR (Synergy Brands Synergy Brands) Premix Ex Taq Kit (TaKaRa). Briefly, the cDNA (complementary DNA) was used as the template for qPCR detection using TaqMan Technology on an Applied Biosystems 7000 sequence detection system (Applied Biosystems, Foster City, CA). Then we detected the expression of YAP1, SOX2 (SRY-related HMG-box 2), CD133, OCT4 (Octamer-binding transcription factor-4) and NANOG genes by using commercially available primer and probe sequences (Applied Biosystems).

\section{miRNA transfection}

The miRNA mimics were synthesized through the Ribobio Technology. Both the H520 and HCC95 cells were grown in 6-well plates and transfected with Lipofectamine 3000 (Invitrogen; Thermo Fisher Scientific) in Opti-MEM (Invitrogen; Thermo Fisher Scientific). After $48 \mathrm{~h}$, cells were harvested to the following experiments.

\section{Luciferase activity assay}

The luciferase assay was performed using the dualLuciferase ${ }^{\circledR}$ reporter assay kit (Promega, Madison, WI). Briefly, the 293T cells were grown in 24-well plates and co-transfected with miR-NC or miR-214-3p mimics, pmirglo-H-YAP1-3' Untranslated Region (UTR) wide type (WT) or pmirglo-H-YAP1-3'UTR-mutation type (Mut) or pGL3-YAP1 promoter, and renilla luciferase vector with Lipofectamine 2000 (Invitrogen). After 36 h, the firefly luciferase activity normalized to the renilla luciferase.

\section{CCK8 assay}

Using the Cell Counting Kit-8 (CCK8) (Dojindo, Kumamoto, Japan), cell proliferation and viability were measured. After transfection, cells were grown into 96-well plates (1000 cells/well, $\mathrm{N}=4)$. The optical density value (OD value) at $450 \mathrm{~nm}$ of the cells at the specified time was recorded. 

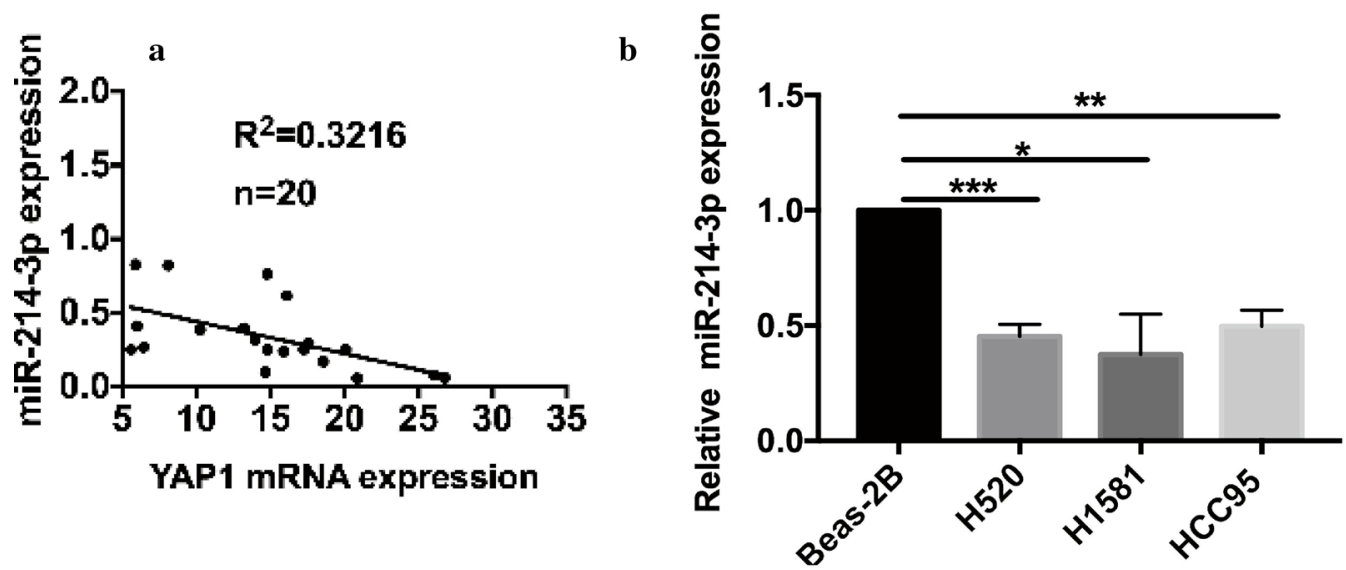

c

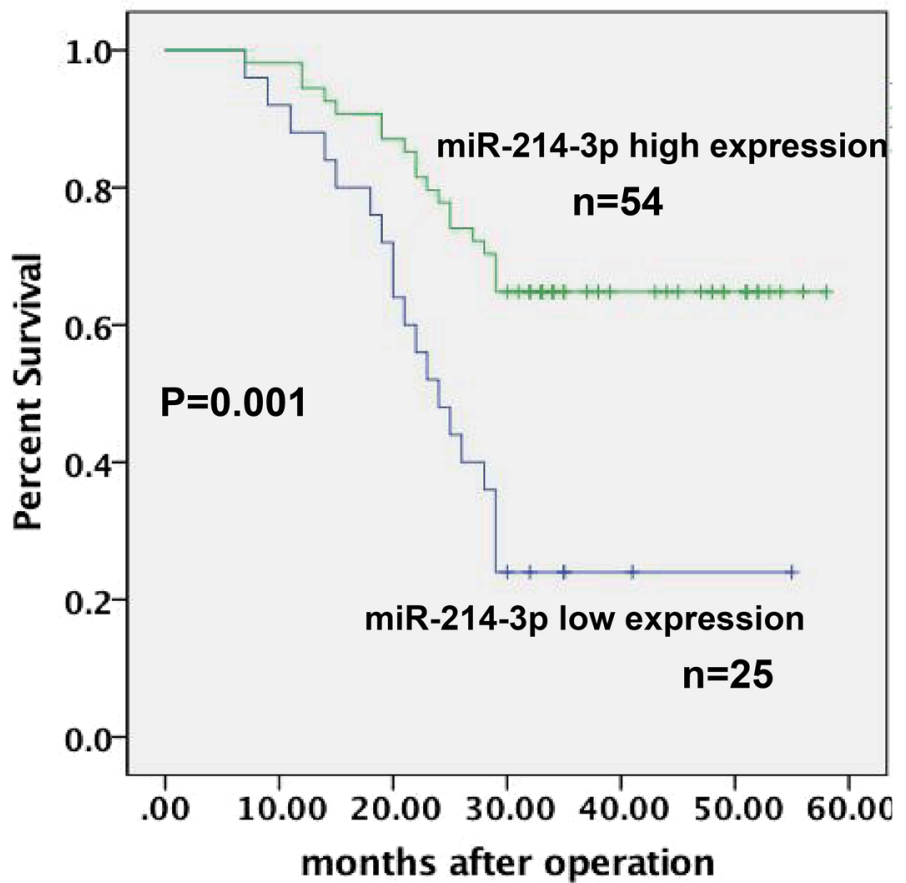

Fig. 1 In lung squamous cell cancer (LSCC), the MiR-214-3p is significantly downregulated, which is related to better clinical outcomes. a Correlation analysis of miR-214-3p with YAP1 levels, as detected by qPCR. $(n=20)$. $\mathbf{b}$ Relative miR-214-3p levels in LSCC cell lines and the normal bronchial epithelial cell line BEAS-2B was used as the control. c Kaplan-Meier analysis of overall survival, relying on miR-214 levels in 79 LSCC patients, showed patients with higher miR-214 levels possessed a more favorable clinical outcome than patients with lower miR-214 levels $(P=0.001)$

\section{Colony-forming assay}

All 500 cells with different treatments were grown into 6-well plates. The cells were cultured for at least 10 days until the colonies became visible and noticeable. The cells were washed with phosphate buffer saline (PBS), fixed with $4 \%$ polyformaldehyde (Sigma-Aldrich, St. Louis, MO), and then stained with Giemsa (Solarbio, Beijing, China). Image $J$ recorded photos of the colonies.

\section{Sphere formation assay}

Briefly, adherent H520 and HCC95 cells with different treatments were seeded into the 6-well ultra-low attachment plates at a density of 3000/well. After 14 days, spheres (> $75 \mu \mathrm{m}$ diameter) were tallied and captured. Every 3 days, the culture medium was refreshed, and oncosphere numbers were counted using the Leica digital camera. The serum-free DMEM-F12 medium (Life Technologies, Grand Island, NY) involves the B-27 


\begin{tabular}{|c|c|c|c|c|}
\hline \multirow[t]{2}{*}{ Variable } & \multirow[t]{2}{*}{$\mathbf{n}$} & \multicolumn{2}{|l|}{ miR-214-3p } & \multirow[t]{2}{*}{$\mathbf{P}$} \\
\hline & & Low $(n=25)$ & High $(n=54)$ & \\
\hline \multicolumn{5}{|l|}{ Age (years) } \\
\hline$<65$ & 42 & 12 & 30 & \multirow[t]{2}{*}{0.531} \\
\hline$\geq 65$ & 37 & 13 & 24 & \\
\hline \multicolumn{5}{|l|}{ Gender } \\
\hline Female & 41 & 16 & 25 & \multirow[t]{2}{*}{0.143} \\
\hline Male & 38 & 9 & 29 & \\
\hline \multicolumn{5}{|l|}{ Tumor size (cm) } \\
\hline$\leq 4.5$ & 39 & 8 & 31 & \multirow[t]{2}{*}{0.036} \\
\hline$>4.5$ & 40 & 17 & 23 & \\
\hline \multicolumn{5}{|l|}{ Smoke } \\
\hline Yes & 31 & 6 & 25 & \multirow[t]{2}{*}{0.059} \\
\hline No & 48 & 19 & 29 & \\
\hline \multicolumn{5}{|l|}{ TNM stage } \\
\hline$|/| \mid$ & 59 & 15 & 44 & \multirow[t]{2}{*}{0.041} \\
\hline III/IV & 20 & 10 & 10 & \\
\hline \multicolumn{5}{|c|}{ Differential degree } \\
\hline Poorly & 52 & 20 & 32 & \multirow[t]{2}{*}{0.071} \\
\hline Well, moderate & 27 & 5 & 22 & \\
\hline
\end{tabular}

Low/high by the sample median. Pearson $X^{2}$ test ${ }^{*} p<0.05$ was considered statistically significant

Supplement (Life Technologies, Grand Island, NY), $20 \mathrm{ng} / \mathrm{ml}$ basic fibroblast growth factor (bFGF, Gibco), and $20 \mathrm{ng} / \mathrm{ml}$ Epidermal Growth Factor (EGF, Gibco).

\section{Immunofluorescence method}

All paraffin-sections of mouse lung tumors were acquired from a Leica Cryostat. The tumor sections were incubated with one of the following polyclonal antibodies: rabbit YAP1 antibody, rabbit SOX2 antibody, and rabbit CD133 antibody (all at 1:100 dilution, CST, Danvers,
MA). After washing with the PBS for at least three times, the sections were incubated with the Alexa-488-conjugated or Alexa-594-conjugated secondary anti-body (1:200 dilution, Life Technologies) for $1 \mathrm{~h}$ at room temperature. Furthermore, the nuclei were stained with 4',6-diamidino-2-phenylindole (DAPI) (1:500 dilution, Beyotime Institute of Biotechnology, China). All images were taken using a confocal laser-scanning microscope (Leica TCS SP5 II, Germany).

\section{Tumor Xenograft Model}

Lung squamous cells transfected with miR-NC mimics or miR-214-3p mimics were triple-washed in the PBS and then suspended in matrigel. Cells were subcutaneously injected into the armpit of each mouse. Every 2 days, each tumor diameter was measured. Tumor volume was calculated by the equation: tumor volume $(\mathrm{mm} 3)=1 / 2 \times$ length $(\mathrm{mm}) \times$ width $\mathrm{mm} 2$. After nearly 4 weeks, tumors were all collected for subsequent analysis. All male BALB/C nude mice (SLAC, Shanghai) were fed at the specific pathogen free (SPF) Animal Center of Shanghai Chest Hospital. All experiments were performed according to the guidelines.

\section{Orthotropic lung tumor model}

Cells were suspended in matrigel and orthotropically injected into the left lateral lungs of the mice, as described with minor modifications.

\section{Statistical analysis}

GraphPad Prism software was employed for statistical analysis. All results were presented as mean \pm Standard error of the mean (SEM) where indicated formats least three independent replicate experiments. Statistical differences were investigated using the Student's t-test, $x^{2}$ test, and repeated measures analysis of variance. The

Table 2 Univariate and multivariate analysis of different prognostic variables and overall survival (OS) in 79 LSCC patients

\begin{tabular}{|c|c|c|c|c|}
\hline \multirow[t]{2}{*}{ Variables } & \multicolumn{2}{|l|}{ Univariate analysis } & \multicolumn{2}{|c|}{ Multivariate analysis model } \\
\hline & $\mathrm{HR}(95 \% \mathrm{Cl})$ & $\mathbf{P}$ & $\mathrm{HR}(95 \% \mathrm{Cl})$ & $\mathbf{P}$ \\
\hline Age (<65 vs $\geq 65)$ & $0.663(0.350-1.257)$ & 0.208 & & \\
\hline Gender (Female vs male) & $0.851(0.450-1.608)$ & 0.619 & & \\
\hline Tumor size ( $\leq 4.5$ vs $>4.5$ ) & $0.235(0.111-0.498)$ & $<0.001$ & $0.383(0.158-0.928)$ & 0.034 \\
\hline Smoke (yes vs no) & $1.245(0.654-2.372)$ & 0.504 & & \\
\hline TNM stage (I/II vs III/IV) & $0.235(0.123-0.448)$ & $<0.001$ & $0.508(0.234-1.104)$ & 0.087 \\
\hline Differentiation degree (well/moderate vs poorly) & $2.316(1.223-4.387)$ & 0.010 & $1.520(0.712-3.246)$ & 0.280 \\
\hline miR-214-3p expression (low vs high) & $0.331(0.175-0.628)$ & 0.001 & $0.489(0.249-0.960)$ & 0.038 \\
\hline
\end{tabular}




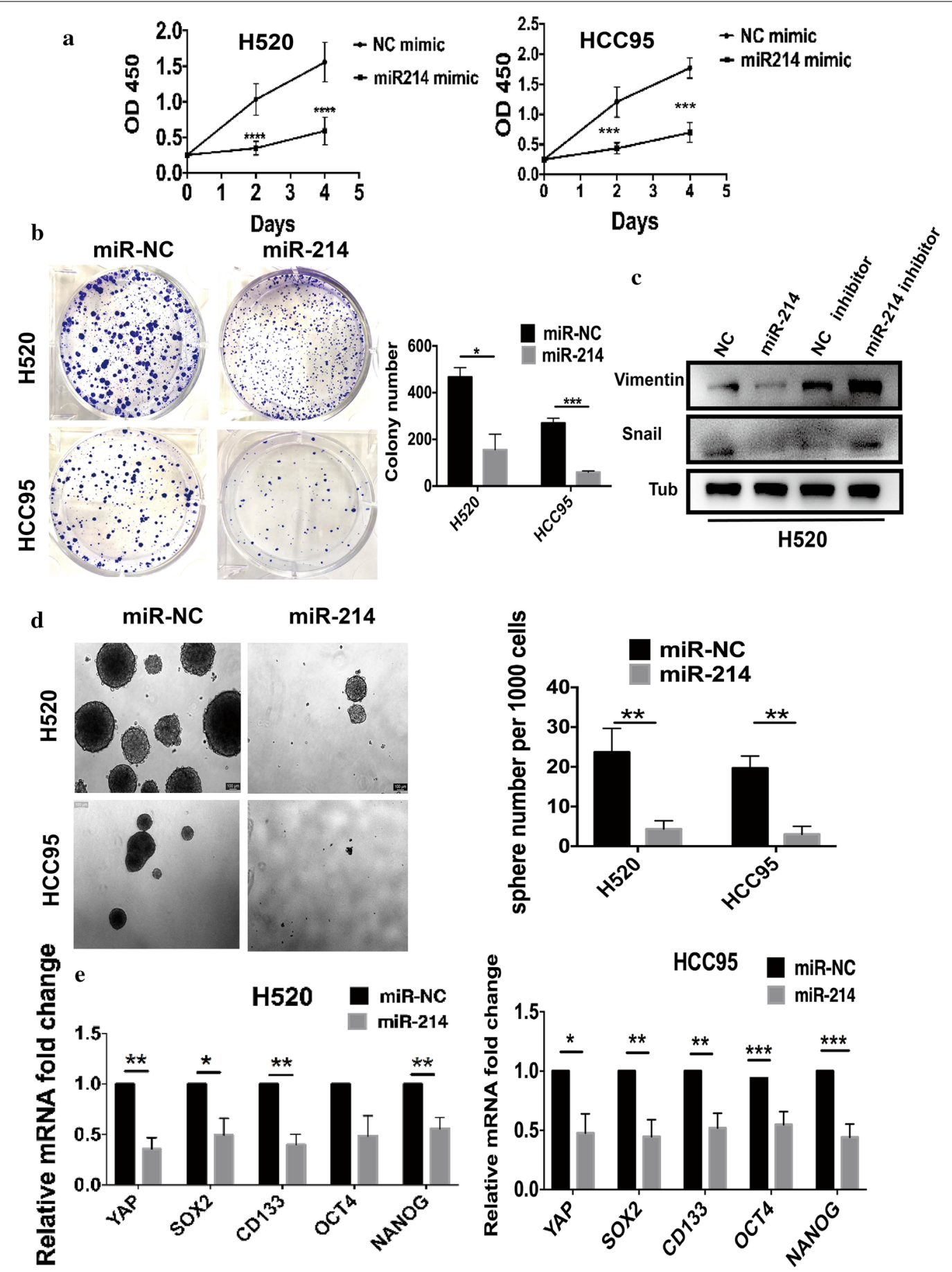

Fig. 2 MiR-214-3p overexpression impairs LSCC proliferation and CSC properties in vitro. a CCK-8 assay indicated that MiR-214-3p reduced cell proliferation. $\mathbf{b}$ Colony-forming assays showed the effect of miR-214-3p. c Protein levels of Vimentin and Snail after overexpression or knockdown of miR-214-3p. $\mathbf{d}$ Sphere forming assays were used to examine the effects of miR-214-3p OE on LSCC. e The qPCR showed the expression of stem-related genes in both cells. Data are represented as mean and SEM from three independent experiments. ${ }^{*} P<0.05$. ${ }^{* *} P<0.01$. ${ }^{* * *} P<0.001$

Kaplan-Meier method was performed to assess overall survival (OS), and the log-rank test was performed to analyze the curves. Finally, the Cox model was utilized to identify independent prognostic factors. The statistical significance was set at $\mathrm{P}<0.05$. 


\section{Results}

MiR-214-3p is downregulated in LSCC and is significantly associated with better clinical prognosis

While YAP1 is elevated, the miR-214-3p is downregulated in tumor species compared to the matched normal bronchial epithelium using q-PCR analysis [19, 20]. In this context, our further analysis found a robust negative correlation between the miR-214-3p expression and YAP1 mRNA expression in LSCC species $(n=20$, Fig. 1a). Next, we detected the miR-214-3p RNA levels in some LSCC cell lines and we observed the reduction of miR-214-3p in LSCC cell lines normalized to the normal bronchial epithelial cell line BEAS-2B accordingly (Fig. 1b). Then, we explored the relationship between miR-214-3p and corresponding clinical features. Patients were divided into two groups according to the median level of the miR-214-3p expression. We found the association of high miR-214-3p expression to smaller tumor size and lower Tumor Node Metastasis (TNM) stage (Table 1). There was no significant relationship between miR-214-3p and other clinical features, such as age and gender. Furthermore, Kaplan-Meier (KM) survival curves indicated that patients with high miR-214 expression possessed better clinical outcomes $(\mathrm{P}=0.001$, Fig. $1 \mathrm{c}, \mathrm{n}=79)$. The Cox regression analysis model revealed that the miR-214 expression and tumor size were independent prognostic indicators for LSCC (Table 2). In summary, these results suggested that miR214-3p could have a critical role in LSCC initiation and progression.

\section{Increased miR-214-3p inhibits LSCC cell proliferation and CSC properties in vitro}

To test the effects of miR-214-3p in lung cancer, we designed subsequent experiments. First, CCK-8 6assay demonstrated that the miR-214-3p overexpression (miR214-3p OE) particularly inhibited proliferation in $\mathrm{H} 520$ and HCC95 cells compared to the NC groups (Fig. 2a). Vimentin and Snail are mesenchymal markers involved in many aspects of cellular organization, signaling, and proliferation. Western-bolt analysis proved that the miR-214-3p OE reduced the protein levels of Vimentin and Snail, while the miR-214-3p knockdown increased Vimentin and Snail protein levels accordingly (Fig. 2c). Additionally, the miR-214-3p OE remarkably inhibited colony and sphere formations compared with the control group (Fig. 2b and d). Moreover, q-PCR proved that the miR-214-3p OE substantially decreased the RNA expression of CSC-specific markers involving CD133, SOX2, OCT4, and NANOG (Fig. 2e). In addition, we detected the proteins level of CSC-specific markers, and the same conclusion was exhibited in Additional file 2: Figure S2.

\section{MiR-214-3p inhibits CSC properties of LSCC in vivo and decreases YAP1 protein level}

Based on the above findings regarding in vitro, we illuminated the role of miR-214-3p in vivo. Then, H520 and HCC95 cells transfected with miR-214-3p or NC mimics were administered into flanks of nude mice. Figures $3 \mathrm{a}-\mathrm{f}$ exhibited how tumor volume and weight in the miR214-3p OE group were smaller than the NC group in both H520 and HCC95 mice models. Fluorescence confocal microscope analysis implied that miR-214-3p OE significantly decreased YAP1 levels in mouse models (Fig. $3 g$ and $\mathrm{h}$ ). The Hippo pathway regulates proliferation and apoptosis to control tissue size. Also, we observed that the miR-214-3p increased YAP1 phosphorylation and decreased YAP1 levels (Fig. 3i). Altogether, the results explained above demonstrated how miR-214-3p OE suppressed LSCC tumor progression by targeting YAP1.

To further thoroughly verify the physiological function of miR-214-3p, we developed orthotropic mice models, which closely recapitulated human lung cancer. Cells were orthotropically administered into the left lateral lungs of nude mice. Compared to the NC groups, the miR-214-3p OE remarkably decreased tumor occurrence and tumor size (Fig. $4 \mathrm{a}$ and b). Figure $4 \mathrm{c}$ illustrated that mouse body weight in the miR-214-3p OE group is heavier than the NC group after injection, mainly caused by cachexia. Furthermore, the miR-214-3p OE significantly prolonged OS than the control group (Fig. 4d). In the future, larger animal models are needed for further research. Immunofluorescence (IF) staining assays showed a decrease of YAP1 and stem-related biomarkers, including SOX2 and CD133. This suggests the observable loss of proliferation and CSC properties in tumors (Fig. $4 \mathrm{e}-\mathrm{g}$ ).

\section{The miR-214-3p directly interacts with YAP1 in LSCC cells} Given that the miR-214-3p is downregulated while YAP1 is up-regulated in LSCC patients, we speculated that YAP1 could be a target of miR-214-3p. Bioinformatic

\footnotetext{
(See figure on next page.)

Fig. 3 miR-214-3p impairs tumorigenicity of LSCC in vivo and activates the Hippo pathway decreases YAP1 protein level. a and $\mathbf{b}$ H520 and HCC95 cells transfected with miR-214-3p were injected into nude mice. c and $\mathbf{e}$ Tumor weight and tumor volume were analyzed in H520 cells. $\mathbf{d}$ and $\mathbf{f}$ Tumor weight and tumor volume were analyzed in HCC95 cells. $\mathbf{g}$ and $\mathbf{h}$ Fluorescence confocal microscope analysis demonstrated miR-214-3p overexpression decreased YAP1 levels of mice tumors. i Protein levels of Hippo components, after transfected with miR-214-3p in H520 cells. (At first, 7 mice models were established in each group, but 2 mice died in HCC95 groups). $p<0.05,{ }^{* *} p<0.01,{ }^{* * *} p<0.001$
} 
a

H520

miR-214-3p

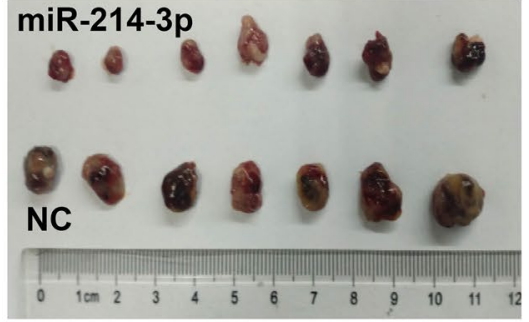

c

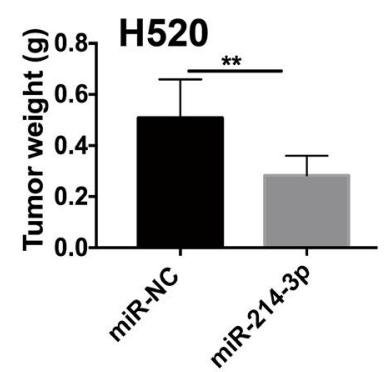

e
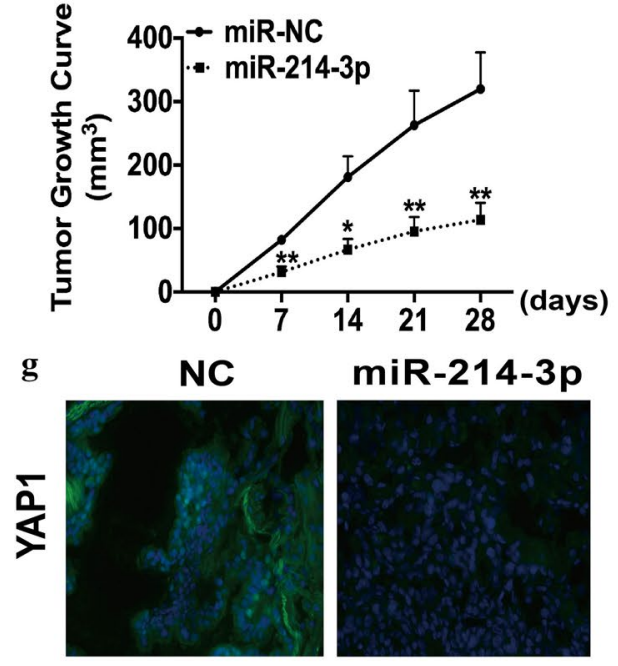

H520 b

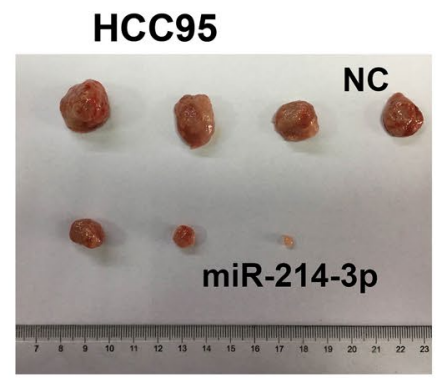

d

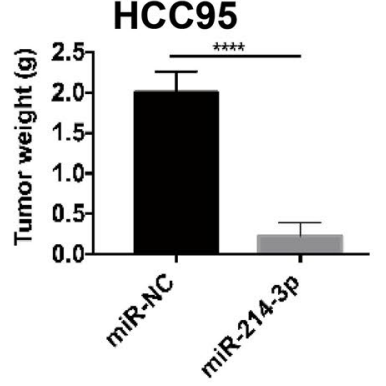

$\sum_{0}^{0}$

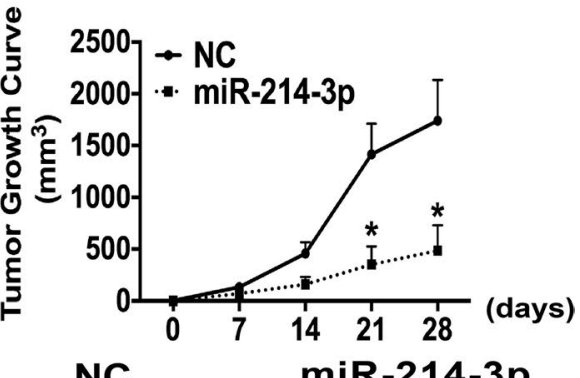

$\frac{1}{5}$

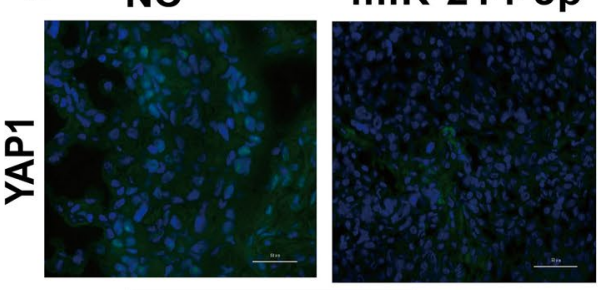

HCC95

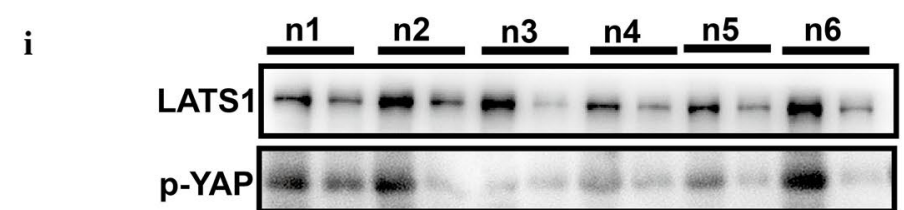

옥

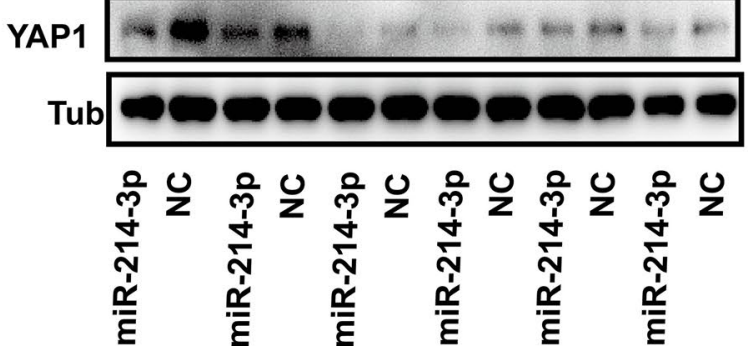


a

H520
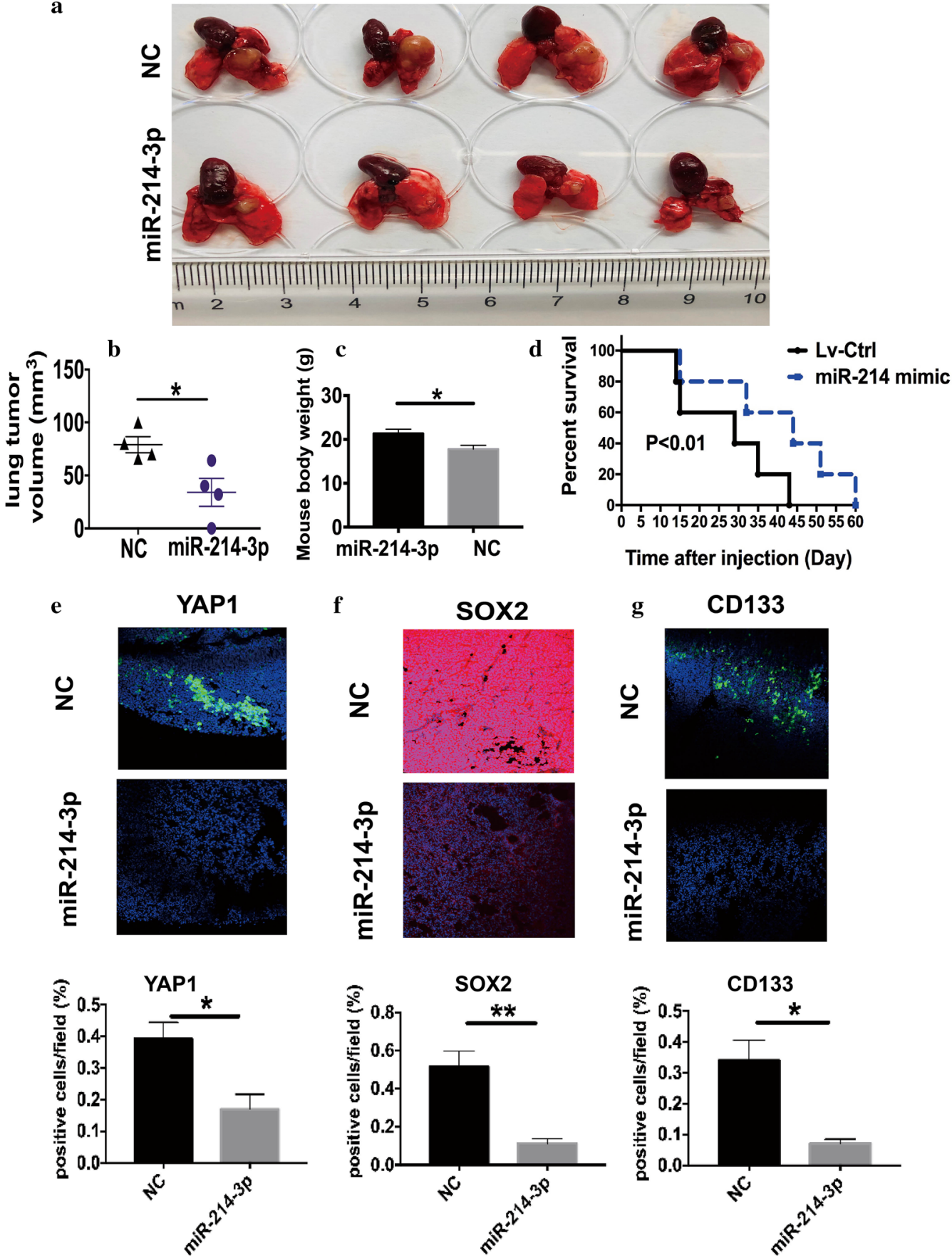

Fig. 4 MiR-214 overexpression impairs LSCC tumor initiation and progression of orthotropic models. a Images of primary tumors in the left lungs at day 21 from each group after injection of H520 cells transfected with miR-214-3p mimics and NC mimics. $\mathbf{b}$ and $\mathbf{c}$ Mean primary tumor volume and body weight from two groups. (Error bars represent SEM; ${ }^{*} p<0.05$ ). $\mathbf{d}$ Survival for the mice in each group evaluated. ${ }^{* *} p<0.01$. e-g Upper panel: Representative IF images of YAP1 and stem-related biomarkers, including SOX2 and CD133. Lower panel: Quantification of biomarker expression (Error bars represent SEM, $N=6$; ${ }^{*} \mathrm{p}<0.05$; ${ }^{* *} \mathrm{P}<0.01$ ) 
a

YAP1-3'UTR-wt 3'-TTTGTTG-5'

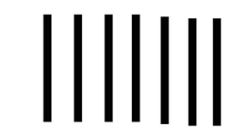

miR-214-3p 5'-AAACGGC-3'
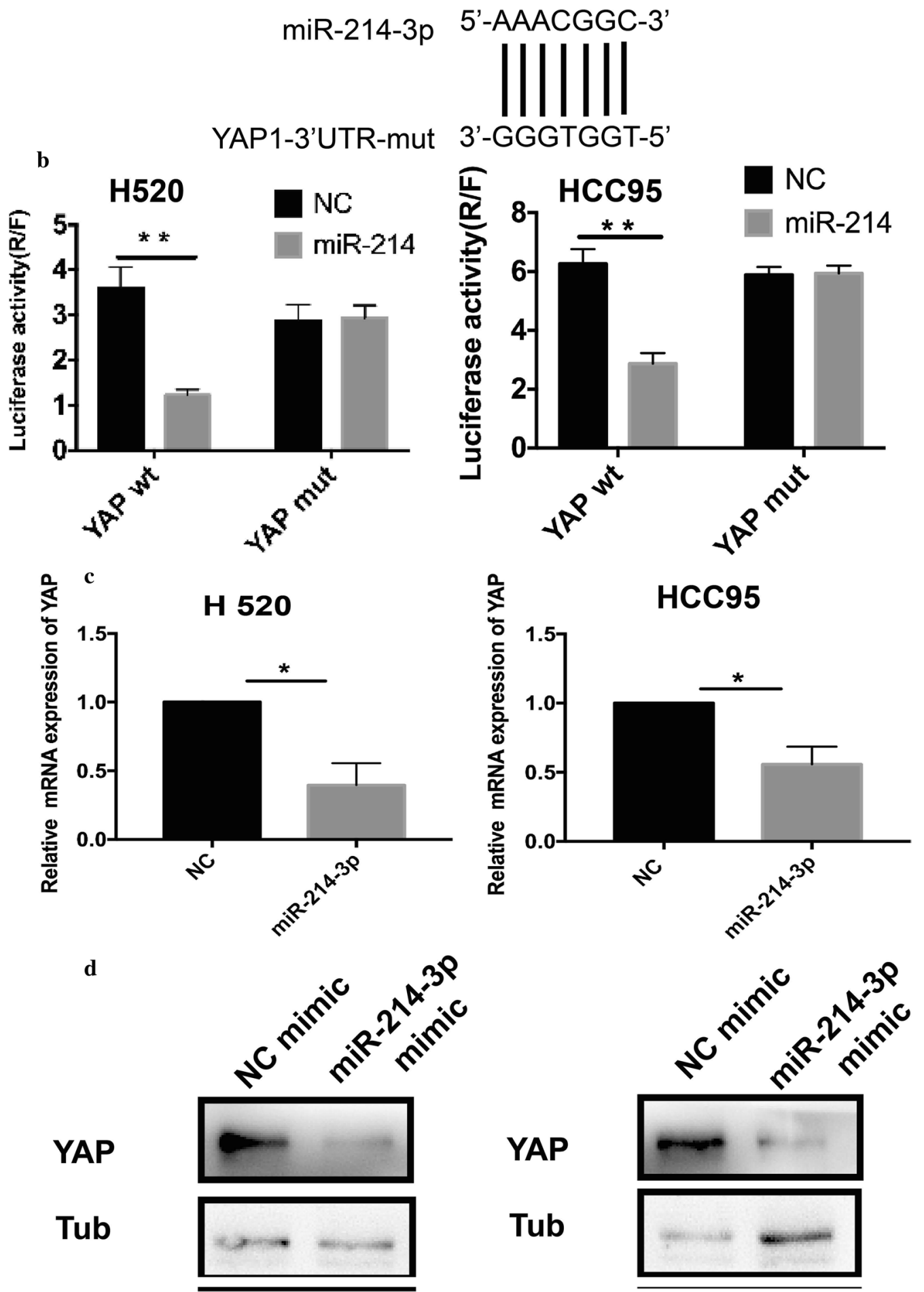

Fig. 5 MiR-214-3p directly targets YAP1 in LSCC cells. a Binding sites of miR-214 in the YAP1 3'UTR. $\mathbf{b}$ Luciferase activity of each combination was assessed. $\mathbf{c}$ The YAP1 mRNA levels in H520 cell lines. $\mathbf{d}$ The YAP1 protein levels in LSCC cell lines transfected with miR-214 mimics. ${ }^{*} p<0.05$, $\left.{ }^{* *} p<0.01\right)$ 
(See figure on next page.)

Fig. 6 Rescue experiments confirm that YAP1 liberated the growth and CSC properties repressed by miR-214. miR-214-3p represses growth and CSC properties by inhibiting YAP1. a CCK experiments in LSCC cells transfected with different plasmids. $\mathbf{b}$ Colony formation in cells transduced with different plasmids. c Sphere-forming experiments in LSCC cells transfected with different plasmids. $\mathbf{d}$ Re-introduction of YAP1 rescued the reductions of CSC-specific markers in both cell lines. $\mathbf{d}$, e. Vimentin and Snail protein levels were detected in LSCC cells transfected with different plasmids. e, f. In subcutaneous mice models, the re-expression of YAP1 remarkably decreased the anti-tumor effects of miR-214-3p

algorithms such as miRanda and TargetScan recommended that YAP1 possesses potential complementary sites for the miR-214-3p seed region in the $3^{\prime}$ Untranslated Region (3'UTR) (Fig. 5a). To verify this hypothesis, we transfected both the H520 and HCC95 cells with miR214 or miR-214 inhibitors, and YAP1 mRNA and protein levels were measured. Results showed that the miR-214 OE significantly decreased YAP1 mRNA and protein levels in both cell lines, while miR-214 knockdown (KD) led to increased YAP1 expression (Fig. 5c and d). Furthermore, to determine the direct binding site between the miR-214-3p and YAP1, we performed a luciferase assay. Then, we constructed the wild type of and mutated seed sequences in the $3^{\prime}$ UTR of YAP1 mRNA. The YAP1wt-3'UTR and its mutant type (YAP1-mt-3'UTR) were amplified and cloned downstream of a luciferase reporter gene in the pGL3-basic vector. The luciferase reporter experiment revealed that the co-transfection of pcDNAmiR-214 and YAP1-wt-3'UTR in H520 and HCC95 cells remarkably inhibited luciferase activity compared to the NC cells (Fig. 5b). However, there was no difference in the luciferase activity in cells that co-transfected with YAP1-mt-3'UTR and pcDNAmiR-214 (Fig. 5b). Therefore, we confirmed that miR-214-3p directly targets YAP1 by specifically binding on the $3^{\prime}$ UTR of YAP1 in LSCC cell lines.

\section{Rescue experiments illustrated that YAP1 liberated the growth and CSC properties inhibited by miR-214}

These findings encouraged us to explore whether the miR-214-3p repressed LSCC growth and CSC properties via YAP1. Our previous work revealed that YAP1 KD remarkably decreased sphere and colony formations and aldehyde dehydrogenase gene (ALDH) proportion. Preveiously, our group have proved that YAP1 KD substantially reduced stem-related genes levels of lung cancer cells [19]. These results suggested that YAP1 KD could mimic the role of miR-214-3p OE. Our findings above confirmed that silenced YAP1 led to the same phenotypes as miR-214-3p OE did, implying that miR-214-3p inhibited proliferation and CSC phenotype via YAP1. To validate this hypothesis, we performed a subsequent rescue experiment in H520 and HCC95 cells. Cells transfected with miR-214 were transduced with a pcDNA3.1-YAP1 plasmid. Interestingly, YAP1 OE partially rescued the proliferation capacity inhibited by the miR-214 (Fig. 6a).
The YAP1 plasmid transduction liberated the inhibited growths of miR-214-3p OE colonies and spheres (Fig. 6b and c). We also found that re-introduction of YAP1 rescued the reductions of CSC-specific markers in both H520 and HCC95 cell lines (Fig. 6d). In addition, the reintroduction of YAP1 rescued the reductions of Vimentin and Snail protein expressions caused by the miR-214-3p OE (Fig. 6e). Furthermore, in mice models, the restoration of YAP1 expression significantly liberated the decreased proliferation and self-renewal abilities caused by the miR-214-3p OE in LSCC (Fig. 6f).

\section{Discussion}

This study confirmed that YAP1 was overexpressed while miR-214-3p was downregulated in LSCC tissues, which was inversely correlated with each other. The KM survival curves indicated that patients with high miR-214 expression possessed better clinical outcomes than those with low expression. In this case, we initially reported that the miR-214-3p strongly modulated CSC phenotypes of LSCC in vitro and in vivo. We provided robust evidence on how miR-214-3p served as a tumor suppressor and a promising target in therapies against LSCC because it imitated and displayed excellent anti-tumor efficacy.

Like many other miRNAs, miR-214 has reportedly been abnormally expressed in various tumors. It plays a dual role by modulating tumor initiation based on the tumor types, either as oncogenes or tumor suppressors. As a tumor suppressor, miR-214 represses tumor progression in hepatocellular, gastric, ovarian, and cervical cancers. However, it is also reported to promote tumor migration in melanoma [21-25]. Furthermore, miR-214 acts as either an oncogene or a suppressor, depending on the genetic background or the cancer type. Interestingly, in lung cancer, Wu et al. reported that miR-214 OE significantly diminished the migration and invasion of non-small cell lung cancer (NSCLC) cells, while Long et al. found that miR-214 expression contributed to epithelial-mesenchymal transition (EMT) and metastasis of lung adenocarcinoma $[26,27]$. However, the impact and regulatory mechanisms of miR-214 in LSCC remain to be extensively explored.

We demonstrated the role of miR-214 as a tumor suppressor and how miR-214 impaired the CSC properties of LSCC by gain- and loss-of-function assays. To our knowledge, the crucial role of miRNA is to regulate the 


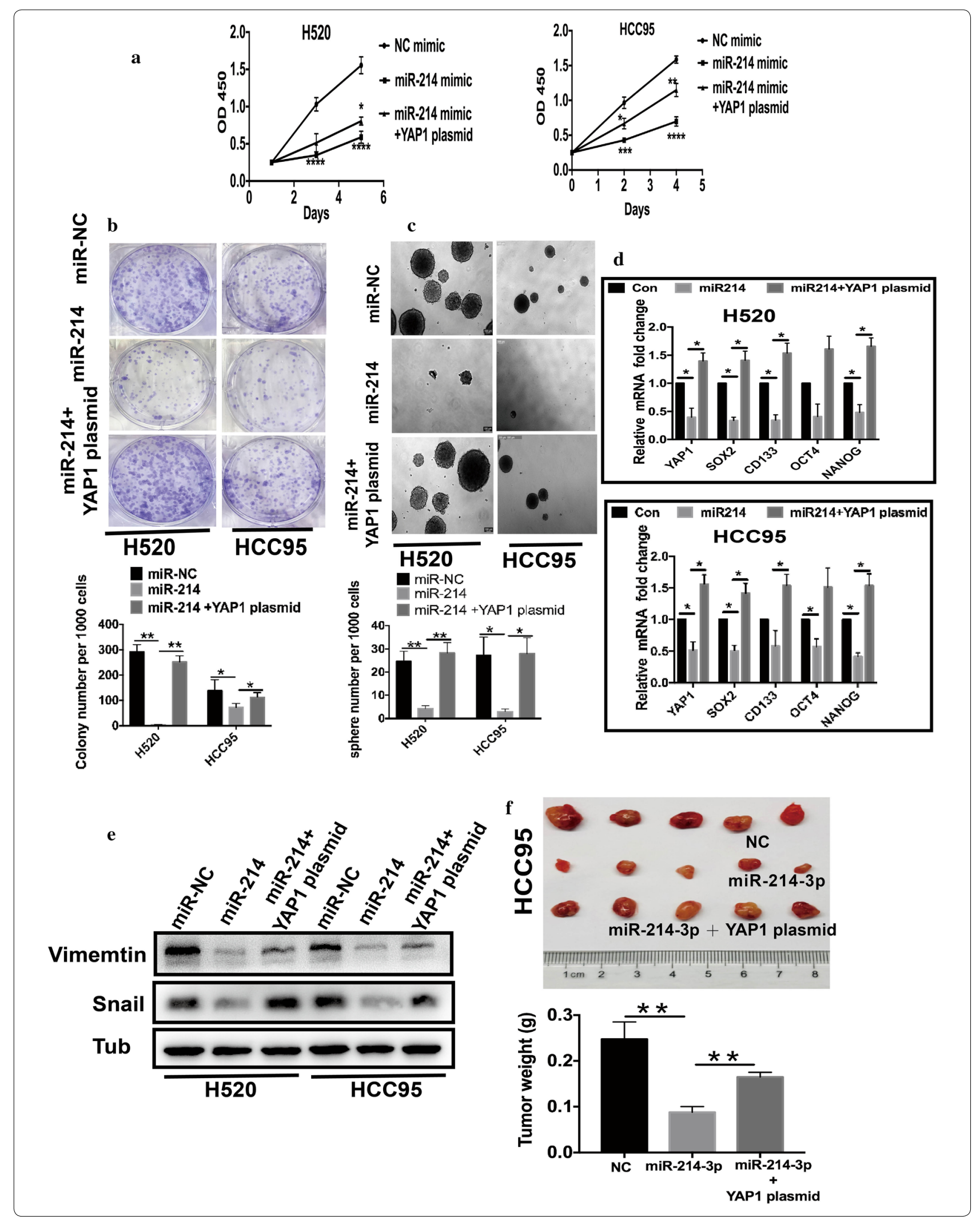


expression of its downstream genes through the mRNA cleavage and/or by inhibition of translation, depending on the extent of complementarity with the $3^{\prime} \mathrm{UTR}$ of target genes. Our group previously confirmed that the miR-214-3p was downregulated, whereas YAP1 was upregulated in LSCC tissues [19]. In this context, we reported that miR-214 was inversely correlated with YAP1, which stimulated our attention and interest. Further investigations validated our hypothesis that YAP1 was a direct downstream gene of miR-214 in LSCC. First, miR-214 OE evidently reduced YAP1 mRNA and protein expression in LSCC. Second, ectopic miR-214 levels significantly impaired a luciferase reporter's activity, which contains the $3^{\prime} U T R$ sequence of YAP1. Third, rescue experiments showed that YAP1 re-expression abrogated the anti-tumor effect of miR-214 on LSCC to a certain extent.

To conclude, miR-214 acts as a critical regulator of CSC properties in LSCC. We also reported that miR214 inhibited LSCC proliferation and CSC capacities via targeting YAP1 to activate the Hippo signaling pathway. The identification of tumor-specific miRNAs and target genes is necessary to understand the underlying mechanisms of LSCC initiation. This is of great value to design new strategies. In the future, miR-214-3p might become a promising prognostic marker. Finally, targeting the miR214-YAP1 axis could be identified as a promising strategy in LSCC.

\section{Conclusion}

Our data revealed that miR-214 could inhibit LSCC cell proliferation through the Hippo signaling pathway. Collectively, these findings provide new insight into the role of miR-214, which might serve as a novel therapeutic target for LSCC treatment.

\section{Supplementary information}

Supplementary information accompanies this paper at https://doi. org/10.1186/s12935-020-01506-2.

Additional file 1. MicroRNA expression profile in LSCC human samples.

Additional file 2. MiR-214-3p OE substantially decreased the proteins level of CSC-specific markers.

\section{Abbreviations}

miRNAs: microRNAs; LSCC: Lung squamous cell cancer; YAP1:Yes-associated protein 1; CSCs: Cancer stem-like cells; LC: Lung cancer; AC: Lung adenocarcinoma; Mst1/2: Mammalian Sterile20-like Kinase1/2; Sav1: Salvador; FGFR1-amplified: Fibroblast Growth Factor Receptor1-amplified; NC: Negative Control; LATS1: Large tumor suppressor 1; P-YAP1: Phospho-YAP1; RIPA: Radio-Immunoprecipitation Assay; SDS-PAGE gels: Sodium dodecyl sulfatePolyacrylamide Gel Electrophoresis gels; SYBR: Synergy Brands Synergy Brands; CDNA: complementary DNA; SOX2: SRY(sex determination region of Ychromosome)-related HMG-box 2; OCT4: Octamer-binding transcription factor-4; Opti-MEM: Reduced-Serum Medium;WT:Wide type; Mut: Mutation type;
CCK8: Cell Counting Kit-8; OD value: Optical density value; DMEM: Dulbecco Modified Eagle Medium; PBS: Phosphate buffer saline; bFGF: Basic fibroblast growth factor; EGF: Epidermal Growth Factor; DAPI: 4,6-Diamino-2-phenyl indole; SPF: Specific pathogen free; SEM: Standard Error of Mean; OS: Overall survival; TNM: Tumor Node Metastasis; KM: Kaplan-Meier; OE: Overexpression; IF: Immunofluorescence; 3'-UTR: 3'Untranslated Region; KD: Knowdown; ALDH: Aldehyde dehydrogenase gene; NSCLC: Non-small cell lung cancer; EMT: Epithelial-mesenchymal transition.

\section{Acknowledgements \\ Not applicable.}

\section{Authors' contributions}

$T L$ and $S L$ designed the study; $T L$ and $Y Y$ performed the experiments and prepared the figures; $T L, Y Y, Z L$ contributed to drafting the manuscript. All authors read and approved the final manuscript.

\section{Funding}

This study was supported by The National Key R\&D Program of China (2016YFC1303300 to S. Lu), Shanghai Chest Hospital Project of Collaborative Innovation(YJXT20190105,YJXT20190209 to S.L and Z.L.), the National Natural Science Foundation of China (81672272 to S. Lu and 81773115 to W. Xia), the National Key Grant of China (2016YFC0906400 to W. Xia), Wu Jie Ping Medical Foundation (320.6750.13350), the Key Project of Shanghai Health \& Family Planning (201540365 to S. Lu), Shanghai Municipal Science \& Technology Commission Research Project (17431906103 to S. Lu), Shanghai Scientific Research Projects (14140902800 to S. Lu), and AstraZeneca Research Funding. Clinical Research Plan of SHDC (16CR3005A to S. L.), Shanghai Science and Technology Commission guidance projects(18411968200 to Z.L.), MedicalEngineering Joint Funds of Shanghai Jiao Tong University YG2017MS81 (to Z.L.), and Shanghai Youth Top Talent Project (to Z.L.)

\section{Availability of data and materials}

The datasets used and/or analyzed during the current study are available from the corresponding author on reasonable request.

\section{Ethics approval and consent to participate}

The study was approved by the Ethics Review Committee of Shanghai Chest Hospital (Shanghai, China). The animal study followed the Guidelines for the Animal Care and Use approved by Shanghai Chest Hospital.

\section{Consent for publication}

Not applicable.

\section{Competing interests}

The authors declare that they have no competing interests.

Received: 11 May 2020 Accepted: 18 August 2020

Published online: 27 August 2020

\section{References}

1. Torre LA, et al. Global cancer statistics, 2012. Ca A Cancer J Clin. 2015;65(2):87-108.

2. Bartel DP. MicroRNAs: genomics, biogenesis, mechanism, and function. Cell. 2004;116(2):281-97.

3. Nicoloso MS, et al. MicroRNAs - the micro steering wheel of tumour metastases. Nat Rev Cancer. 2009;9(4):293-302.

4. Calin GA, et al. MicroRNA signatures in human cancers. Nat Rev Cancer. 2006;6(11):857-66.

5. Catalanotto $C$, et al. MicroRNA in control of gene expression: an overview of nuclear functions. Int J Mol Sci. 2016;17(10):1712.

6. Kent OA, et al. A small piece in the cancer puzzle: microRNAs as tumor suppressors and oncogenes. Oncogene. 2006;25(46):6188-96.

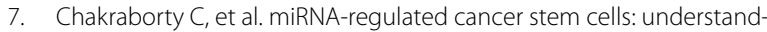
ing the property and the role of miRNA in carcinogenesis. Tumor Biol. 2016;37(10):13039-48.

8. Melton C, et al. MicroRNA regulation of embryonic stem cell self-renewal and differentiation. Cell Biol Stem Cells. 2010;695:105-17. 
9. Liu C, et al. MicroRNA regulation of cancer stem cells. Cancer Res. 2011;71(18):5950-4.

10. Chaudhary AK, et al. Chemosensitization and inhibition of pancreatic cancer stem cell proliferation by overexpression of microRNA-205. Cancer Lett. 2017:402:1-8.

11. Li B, et al. miR-613 inhibits liver cancer stem cell expansion by regulating SOX9 pathway. Gene. 2019;707:78-85.

12. Harvey KF, et al. The Drosophila Mst ortholog, hippo, restricts growth and cell proliferation and promotes apoptosis. Cell. 2003;114(4):457-67.

13. Udan RS, et al. Hippo promotes proliferation arrest and apoptosis in the Salvador/Warts pathway. Nat Cell Biol. 2003;5(10):914-20.

14. Zhao $B$, et al. TEAD mediates YAP-dependent gene induction and growth control. Genes Dev. 2008;22(14):1962-71.

15. Snijders AM, et al. Rare amplicons implicate frequent deregulation of cell fate specification pathways in oral squamous cell carcinoma. Oncogene. 2005;24(26):4232-42.

16. Zhao $B$, et al. Inactivation of YAP oncoprotein by the Hippo pathway is involved in cell contact inhibition and tissue growth control. Genes Dev. 2007;21(21):2747-61.

17. Zhang $X$, et al. The Hippo pathway transcriptional co-activator, YAP, is an ovarian cancer oncogene. Oncogene. 2011;30(25):2810-22.

18. Rizvi S, et al. A hippo and fibroblast growth factor receptor autocrine pathway in cholangiocarcinoma. J Biol Chem. 2016;291(15):8031-47.

19. Lu TT, et al. The Hippo/YAP1 pathway interacts with FGFR1 signaling to maintain stemness in lung cancer. Cancer Lett. 2018;423:36-46.

20. Yang $Y$, et al. Reciprocal regulatory mechanism between miR-214-3p and FGFR1 in FGFR1-amplified lung cancer. Oncogenesis. 2019;8(9):50. https ://doi.org/10.1038/s41389-019-0151-1.
21. Liu Y, et al. MiR-214 suppressed ovarian cancer and negatively regulated semaphorin 4D. Tumor Biol. 2016;37(6):8239-48.

22. Peng $R Q$, et al. miR-214 down-regulates ARL2 and suppresses growth and invasion of cervical cancer cells. Biochem Biophys Res Commun. 2017:484(3):623-30

23. Wang RF, et al. Downregulation of miRNA-214 in cancer-associated fibroblasts contributes to migration and invasion of gastric cancer cells through targeting FGF9 and inducing EMT. J Exp Clin Cancer Res. 2019;38:20.

24. Chen DL, et al. Identification of MicroRNA-214 as a negative regulator of colorectal cancer liver metastasis by way of regulation of fibroblast growth factor receptor 1 expression. Hepatology. 2014;60(2):598-609.

25. Penna $\mathrm{E}$, et al. microRNA-214 contributes to melanoma tumor progression through suppression of TFAP2C. EMBO J. 2011;30(10):1990-2007.

26. Long $H X$, et al. microRNA-214 promotes epithelial-mesenchymal transition and metastasis in lung adenocarcinoma by targeting the suppressorof-fused protein (Sufu). Oncotarget. 2015;6(36):38705-18.

27. Wu DD, et al. Long noncoding RNA MIR31HG is activated by SP1 and promotes cell migration and invasion by sponging miR-214 in NSCLC. Gene. 2019;692:223-30

\section{Publisher's Note}

Springer Nature remains neutral with regard to jurisdictional claims in published maps and institutional affiliations.
Ready to submit your research? Choose BMC and benefit from:

- fast, convenient online submission

- thorough peer review by experienced researchers in your field

- rapid publication on acceptance

- support for research data, including large and complex data types

- gold Open Access which fosters wider collaboration and increased citations

- maximum visibility for your research: over 100M website views per year

At BMC, research is always in progress.

Learn more biomedcentral.com/submissions 